\title{
Organização do Conhecimento e Kant: uma análise do debate epistemológico sobre realismo e idealismo
}

\section{Knowledge Organization of and Kant: an analysis of the epistemological debate on realism and idealism}

\author{
Leila Cristina Weiss | UFSC | leilacw@gmail.com
}

Marisa Bräscher | UFSC | marisa.brascher@gmail.com

Resumo: Estudos epistemológicos podem contribuir para o campo de Organização do Conhecimento (OC). Um importante tema de pesquisa em epistemologia é a distinção entre realismo e antirrealismo ou idealismo. Tal tema recebeu contribuições do filósofo Immanuel Kant, no sentido da definição de uma terceira via para a dicotomia. O presente trabalho tem como propósito refletir sobre o reconhecimento da necessidade de uma terceira via para a dicotomia realismo versus idealismo na OC. Caracteriza-se esse debate em epistemologia, de acordo com Hessen (1980) e Norris (2007), buscam-se as convergências sobre esse debate nas pesquisas sobre as posturas epistêmicas e abordagens na OC, e identifica-se elementos de tal debate nas pesquisas em $O C$ que abordam o pensamento de Immanuel Kant. Considera-se que uma terceira via para a dicotomia realismo versus idealismo na $O C$ seria bem vinda. Pois essa dicotomia da teoria epistemológica se reflete na pesquisa em $\mathrm{OC}$. Um exemplo desse reflexo é o debate sobre abordagem ontológica e epistemológica e postura positivista e pragmática na $O C$. Nesse sentido, $O$ pensamento de Immanuel Kant, em especial sua filosofia transcendental presente na obra 'A crítica da razão pura', pode ser considerado como opção para fundamentar as pesquisas em OC

Palavras-chave: Realismo. Idealismo. Immanuel Kant. Epistemologia. Organização do Conhecimento.

Abstract: Epistemological studies can contribute to the field of Knowledge Organization (KO). An important research topic in epistemology is the distinction between realism and antirealism or idealism. This topic received contributions from the philosopher Immanuel Kant, in the sense of defining a third way to dichotomy. The present work aims to reflect on the recognition of the need for a third way to realism versus idealism dichotomy in $\mathrm{KO}$. It characterizes this debate in epistemology, according to Hessen (1980) and Norris (2007), seeks the convergences on this debate in the research on the epistemological postures and approaches in $\mathrm{KO}$, and identifies elements of such debate in $\mathrm{KO}$ research that approaches the thought of Immanuel Kant. It is considered that a third way to realism versus idealism dichotomy in KO would be welcome. For this epistemological theory dichotomy is reflected in $\mathrm{KO}$ research. Two examples of this reflex, which are naturally linked, is the debate on ontological and epistemological approach and positivist and pragmatic posture in KO. In this sense, the thought of Immanuel Kant, especially his transcendental philosophy present in the work 'The Critique of Pure Reason', can be considered a good option to support the research in $\mathrm{KO}$.

Keywords: Realism. Idealism. Immanuel Kant. Epistemology. Knowledge Organization. 


\section{Introdução}

Existe uma dupla importância de estudos epistemológicos para o campo da Organização do Conhecimento $(\mathrm{OC})$, que diz respeito à fundamentação das pesquisas e métodos no próprio campo e à identificação de pressupostos epistemológicos nos domínios durante o processo de OC.

Hjørland (2003), por exemplo, aponta que o campo de OC não pode ignorar conceitos, teorias e descobertas em disciplinas específicas, e os métodos de OC tem como base os mesmos pressupostos filosóficos que os métodos acadêmicos e científicos. Por isso, segundo o autor, a discussão fundamental das bases da OC está fortemente ligada à discussão de diferentes teorias da epistemologia, mas estudos epistemológicos têm sido raros, existindo uma certa urgência desse tipo de pesquisa no campo de OC para atualizar nossas qualificações nessa área.

Um tópico importante, que apesar de antigo, se faz ainda presente nos debates epistemológicos atuais, é a dicotomia realismo e antirrealismo. Norris (2007), no livro "Epistemologia: conceitos-chave em filosofia", por exemplo, apresenta uma série de debates interligados sobre o realismo versus antirrealismo, externalismo versus internalismo, concepções aléticas versus concepções epistêmicas da verdade, entre outras. Para o autor, tais debates

[...] permitem compreender a base e a implicação dos seus argumentos para questões complexas e importantes. 'Importantes', eu acrescentaria não somente porque têm engajado as mentes de alguns pensadores excepcionalmente brilhantes e qualificados, cujas conclusões seríamos imprudentes se as ignorássemos, mas, também, na medida em que elas se relacionam diretamente com questões de interesse ético e social. (NORRIS, 2007, p.29).

Em epistemologia o debate sobre a dicotomia entre realismo e antirrealismo (que também pode ser denominado de idealismo) encontra uma terceira via no pensamento de Immanuel Kant. De acordo com Hessen (1980), Kant procurou conciliar o realismo com o idealismo, igualmente como o fez entre o racionalismo e o empirismo.

Algumas pesquisas em $\mathrm{OC}$ e Ciência da Informação $(\mathrm{Cl})$ abordam a dicotomia entre realismo e antirrealismo ou idealismo, conforme exposto na seção 2.2 deste trabalho. Apesar disso, dentre as que localizamos, não identificamos qualquer referência ao pensamento de Immanuel Kant. 
Essa pesquisa tem como propósito refletir sobre o reconhecimento da necessidade de uma terceira via para a dicotomia realismo versus antirrealismo na OC. Tem como principal justificativa a relevância dos estudos epistemológicos para a OC, como por exemplo a interrelação entre pensamento e ser, que permeia a discussão sobre realismo e antirrealismo e a discussão sobre diferentes abordagens na OC. A adoção de determinada postura epistêmica influencia o tipo de representação do conhecimento.

Iniciamos descrevendo algumas características desse debate em epistemologia, de acordo com Hessen (1980) e Norris (2007); buscamos as convergências sobre esse debate nas pesquisas sobre as posturas epistêmicas e abordagens na OC; e descrevemos as pesquisas em $O C$ que abordam o pensamento de Immanuel Kant.

\section{Desenvolvimento}

Esta seção é composta por três tópicos, o primeiro foi desenvolvido a partir da análise da literatura de epistemologia e filosofia, o segundo a partir da análise de pesquisas de cunho epistemológico na $\mathrm{OC}$ e $\mathrm{Cl}$ e o terceiro traz a descrição das pesquisas em OC que abordam o pensamento de Immanuel Kant.

\subsection{Realismo, Idealismo e Fenomenalismo}

Hessen (1980), assim como Norris (2007), também aborda o debate epistemológico entre realismo e anti-realismo. Para Hessen (1980) essa questão diz respeito à essência do conhecimento, que possui três tipos de soluções: as prémetafísicas, as metafísicas e as teológicas. Para o autor, as soluções pré-metafísicas, o objetivismo e o subjetivismo, não levam em conta o caráter ontológico do sujeito e do objeto, e as soluções teológicas, monista/panteísta e dualista/teísta, remontam ao último princípio das coisas, ao absoluto, e definindo a partir dele as relações do pensamento e do ser.

Neste trabalho vamos nos ater à discussão das soluções metafísicas, que levam em conta o caráter ontológico do objeto. 
Dentre as soluções, ou poderíamos chamar de respostas, metafísicas sobre a essência do conhecimento, Hessen (1980), aponta o realismo, o idealismo e o fenomenalismo (de Immanuel Kant). O Fenomenalismo seria uma terceira via entre o realismo e o idealismo (que é antirrealista).

Entende-se por realismo a posição epistemológica segundo a qual há coisas reais, independentes da consciência. Este posicionamento epistemológico possui três vertentes: o realismo ingênuo, no qual se considera que as coisas são exatamente como as percebemos e não distingue a percepção e o objeto percebido; no realismo natural, já se admite a distinção entre o conteúdo da percepção e o objeto, mas assim como o realismo ingênuo sustenta que os objetos correspondem exatamente aos conteúdos da percepção; no realismo crítico as propriedades ou qualidades das coisas que apreendemos existem unicamente na nossa consciência e surgem quando determinados estímulos externos atuam sobre nossos órgãos dos sentidos. (HESSEN, 1980)

O idealismo sustenta a tese de que não há coisas reais, independentes da consciência.

suprimidas as coisas reais, só ficam duas classes de objetos, os de consciência (as representações, os sentimentos, etc.), e os ideais (os objetos da lógica e da matemática), o idealismo tem de considerar os pretensos objetos reais como objetos de consciência ou como objetos ideais. Resultam daqui duas formas de idealismo: o subjetivo ou psicológico e o objetivo ou lógico. (HESSEN, 1980, p. 102).

No idealismo subjetivo, considera-se que a realidade está na consciência do sujeito, ou seja, as coisas não são mais do que conteúdos da consciência. $O$ idealismo objetivo tem como ponto de partida a consciência objetiva da ciência, tal como se exprime nas obras científicas. Ou seja, não toma como base um complexo de processos psicológicos, mas sim um conjunto de pensamentos, de juízos. (HESSEN, 1980)

Essas duas posturas epistêmicas divergem sobre qual é o fator determinante para o conhecimento, o sujeito ou o objeto? Immanuel Kant procurou conciliar o realismo com o idealismo, igualmente como o fez entre o racionalismo e o empirismo [1], para isso desenvolveu o fenomenalismo. (HESSEN, 1980). 
O fenomenalismo, como foi desenvolvido por Kant, pode ser resumido a três proposições:

1. A coisa em si é incognoscível. 2. O nosso conhecimento permanece limitado ao mundo fenomênico. 3. Este surge na nossa consciência sensível em relação às formas a priori da intuição e do entendimento. (HESSEN, 1980, p. 111).

Para o fenomenalismo não conhecemos as coisas como são em si, mas como estas se apresentam para nós. Ou seja, há coisas reais, mas não podemos conhecer a sua essência. Coincide com o realismo quando admite coisas reais, mas também coincide com o idealismo quando limita o conhecimento à consciência, ao mundo da aparência, do que resulta imediatamente a impossibilidade de conhecer as coisas em si. (HESSEN, 1980).

Norris (2007) considera que Kant traça uma linha que demarca a fronteira entre questões de natureza epistemológica e questões que têm um caráter estritamente metafísico. $\mathrm{O}$ autor não aponta uma terceira via para o debate sobre o realismo e o anti-realismo em epistemologia, mas afirma que esse debate tem como efeito benéfico focalizar a atenção sobre a existência dos diferentes tipos de proposições. Essas podem ser concebidas, de um lado, como portadoras de valores aléticos (isto é, objetivos), ou de outro, como epistemicamente condicionadas.

Norris (2007) aponta o que denomina de doutrina do realismo transcendental como a melhor opção para o debate sobre 0 realismo e 0 antirrealismo. Inicia a explicação dessa doutrina definindo 'realismo' e 'transcendental'.

Sua definição de realismo é semelhante à definição dada por Hessen (1980), e que já descrevemos. Para a definição de 'transcendental' Norris (2007, p.80) recorre à Kant, afirmando que

se refere às condições de possibilidade do conhecimento e da experiência em geral. Ou seja, ele envolve uma dedução transcendental que explica a nossa capacidade de adquirir esse conhecimento ou ter tal experiência em termos de certos conceitos ou intuições a priori (por exemplo, tempo, espaço e causalidade) que, apenas eles, tornam possível para a mente impor uma ordem inteligível sobre o fluxo sensorial de impressões que, de outra forma, permaneceria apenas incipiente. 
No entanto, Norris (2007) também considera que os dilemas da epistemologia atual remontam à clivagem radical entre o 'idealismo transcendental' e o 'realismo empírico' instaurada por Kant.

Críticas à parte, o que podemos dizer a partir das considerações de Norris (2007) e Hessen (1980) é a inegável contribuição que Kant trouxe ao debate epistemológico sobre realismo e idealismo. E Norris (2007) apesar de trazer críticas ao pensamento de Kant, fundamenta-se nele.

Sobre o pensamento de Kant, Höffe (2005), afirma que a descoberta dos elementos não empíricos e da crítica da razão como meio da sua análise revolucionou o modo tradicional do pensar e conseguiu colocar a filosofia sobre um fundamento seguro. Para o autor, até quem se mantém cético com respeito a tal pretensão de fundamentação deve reconhecer que Kant mudou radicalmente o cenário filosófico.

Um grande número de conceitos e de problemas que se mantém no debate científico e filosófico atual têm sua origem em Kant. $\mathrm{O}$ autor cita como exemplos as noções de conhecimentos a priori e a posteriori, juízos sintéticos e analíticos, argumentos transcendentais, ideias regulativas e constitutivas, $O$ imperativo categórico e a autonomia da vontade. E enfatiza que "as mais diversas orientações do pensamento escolheram Kant como ponto de referência, seja em sentido crítico, seja em sentido afirmativo." (HÖFFE, 2005, p. XXI).

Um exemplo de tais referências que consideramos relevante apontar aqui é a ligação do pensamento de Kant e os estudos da linguagem. O objetivo do ensaio de Muller (2010), por exemplo,

is to stage an intervention by Saussure in Kant's account of the formation and application of empirical concepts. This means at the same time trying out the limits of the claim, variously defended (ECO 1997; APEL 1972/1999; SENDEROWICZ and DASCAL: 1992/1997), that Kant was a linguistic philosopher, however inchoate we may take his semiotics to be. Generally, defense of Kant as a linguistic philosopher takes one implicit thread of the first Critique to be the position that "there are no judgments without language"(BENNETT 1966: 87), and I defend a version of this position in light of a productive comparison of Kantian concept formation and Saussure's "language mechanism." (MULLER, 2010, p. 130). 
Outro exemplo é o artigo de Silva (2012), que apresenta um breve diálogo epistemológico entre a teoria do conhecimento de Aristóteles e a crítica filosófica de Kant com as postulações de Saussure acerca da linguagem. Através da exposição teórica de ambos os filósofos, busca situar o pensamento de Saussure a partir do teor binário que ele concede ao estudo da linguagem humana. Por intermédio da concepção de Metafísica, tanto Aristóteles quanto Kant fundamentam grande parte de seus respectivos arcabouços filosóficos e, com base nessa formulação, Silva (2012) traça uma possível conexão com as bases da linguística moderna contidas no Curso de Linguística Geral de Saussure.

\subsection{Posturas epistêmicas na Organização do Conhecimento}

Nas pesquisas de cunho epistemológico na OC é possível identificar algumas convergências com o debate sobre realismo e antirrealismo presente na literatura de epistemologia e que foi brevemente descrito no tópico anterior.

Gnoli (2008), distingue as abordagens na OC em ontológica e epistemológica [2]. De acordo com o autor "Knowledge is both epistemological and ontological, as it passes through human perception by its very nature, but also refers to real objects of the world having some intrinsic structure" (GNOLI, 2008, p. 139).

Ontologia e epistemologia são disciplinas e conceitos da filosofia, e são discutidas pelo autor a partir das perspectivas de Poli $(1996,1997)$, que as distingue da seguinte maneira:

An ontology is not reducible to pure cognitive analysis (in philosophical terms, it is not an epistemology or a theory of knowledge). Ontology represents the 'objective' side ( $=$ on the side of the object), and the theory of knowledge the subjective side (= on the side of the knowing subject) of reality. The two sides are obviously interdependent, but this is not to imply that they are the same ( exactly like the front and rear of a coin). (POLI, 1996, p. 1)

Podemos perceber algumas semelhanças entre a definição dessas abordagens na OC e o debate em epistemologia sobre o realismo e o idealismo.

A primeira semelhança é que assim como acontece nos estudos epistemológicos, existe a dicotomia entre as abordagens na OC. Ou seja, existem 
duas abordagens contrárias que não conversam entre si, conforme descreve Gnoli (2008, p.140)

[...]the philosophy of KO foundations there are two big "schools," which flow parallel in quite independent streams. As we said that knowledge is both ontological and epistemological, some reconciliation between them should probably be sought.

Além disso, no realismo considera-se o mundo objetivo como a essência do conhecimento e o idealismo considera que a essência está ou depende do sujeito, de sua consciência. $O$ que, respectivamente, converge com a noção de ontologia e epistemologia adota por Gnoli (2008).

Weiss e Bräscher (2015) ao analisar comparativamente as propostas de classificação das correntes teórico-metodológicas de Gnoli (2008), que distingue as abordagens na OC em ontológica e epistemológica, e de Hjørland (2007), que distingue os paradigmas na Organização do conhecimento (OC) em pragmático e positivista, observaram que as propostas analisadas foram desenvolvidas a partir de critérios classificatórios distintos: Hjørland (2007) adota as posturas epistêmicas presentes nas pesquisas em OC como critério, enquanto Gnoli (2008) tem como ponto de partida a maneira de ver o objeto de estudo da OC. Apesar dessas diferenças, as autoras consideram as duas propostas de classificação das correntes teórico-metodológicas na OC complementares, pois

Cada postura epistemológica possui critérios de validação do conhecimento próprios e utiliza métodos de pesquisas que visam atender a esses critérios. Isso faz com que um mesmo objeto de pesquisa seja visto de forma distinta, de acordo com a postura epistemológica adotada na pesquisa. Com uma postura epistemológica pragmática se vê o objeto de estudo da OC como epistemológico e com uma postura epistemológica positivista se vê o objeto de estudo da OC como ontológico. (WEISS; BRÄSCHER, 2015, p. 35)

Em Gnoli (2008), é possível encontrar os seguintes exemplos:

In recent decades, Dahlberg $(1974,1978)$ has worked deeply on the theoretical and conceptual foundations of $\mathrm{KO}$ from a philosophical and ontological perspective. Both Dahlberg and Poli have made reference to the philosopher Nicolai Hartmann, who gave new life to ontology in the 20th century. A completely different epistemological approach has been that of domain analysis, recently spreading in information science (Hjørland \& Albrechtsen 1995), which starts KO work by studying how domain specific communities of scholars use terms to denotate concepts. Epistemological knowledge organizers often take examples from language 
and its cultural relativity, and quote philosophers like Wittgenstein and the American pragmatists.

Não localizamos pesquisas sobre os trabalhos de Ingetraut Dahlberg que fizessem referência à questão do realismo ou antirrealismo, mas Francelin e Kobaschi (2011) e Melo e Bräscher (2014) apontam que Dahlberg adota uma postura epistêmica positivista na pesquisa em OC, e Hjørland (2004), indica a postura positivista na $\mathrm{OC}$ como integrante da linha antirrealista e a postura epistêmica pragmática como realista.

Ao tomarmos a posição de Hessen (1980) como referência para comparação, podemos dizer que Hjørland (2004) discute as noções de idealismo subjetivo ou psicológico, idealismo objetivo ou lógico e realismo crítico (ver em seção 2.1 desse trabalho). E desse modo, a postura positivista em OC estaria relacionado ao idealismo subjetivo ou psicológico e a postura pragmática ao idealismo objetivo ou lógico e ao realismo crítico.

Hjørland (2004) apresenta a noção de relevância tradicional como exemplo de idealismo e uma nova proposta para o conceito de relevância, que é considerada por ele como realista.

Tradicionalmente a avaliação dos sistemas de recuperação de informação (SRI) leva em conta, principalmente, os índices de precisão e revocação. Esses índices são calculados a partir da relevância, entendida como o que o usuário já teria em mente, aquilo que o usuário quer encontrar com a busca. Nas palavras do autor:

A given document may be relevant to a given purpose, whether or not the user believes this to be so. Both a reality and beliefs about that reality exist. In information science, most research activities have in recent decades been directed toward user preferences and attitudes, not toward the basis for the knowledge claims represented in information systems. Most relevance research seems to assume that the relevance of given kinds of information can be established by studying the relevance criteria of the users. This is clearly an idealist position, although probably nobody would like to admit that. (HJØRLAND, 2004, p. 497, grifo nosso).

Nesse ponto então, podemos dizer que o autor se refere a um tipo específico de idealismo, o idealismo subjetivo ou psicológico, conforme este é definido por Hessen (1980). 
A outra definição que $\mathrm{o}$ autor defende e considera realista, diz que relevância "is not a subjective phenomenon but rather an objective one. To be engaged in how to identify what is relevant is to be engaged in scientific arguments" (HJØRLAND, 2004, p. 497, grifo nosso).

E indica dois de seus trabalhos anteriores sobre o tema, sendo que em um destes, Hjørland e Christensen (2002, p.964) verifica-se a seguinte definição de relevância "Something (A) is relevant to a task (T) if it increases the likelihood of accomplishing the goal (G), which is implied by T."

Um aspecto dessa definição de relevância é que ela tem como base o argumento da linha utilitarista do pragmatismo de que o verdadeiro significa útil, pois o homem não é essencialmente um ser teórico ou pensante, mas sim um ser prático, um ser de vontade e ação. E desse modo, o conhecimento humano receberia o seu sentido e o seu valor desta sua natureza prática. (HESSEN, 1980).

Tal argumento, de um modo geral, é visto como antirrealista em Norris(2007) e relacionado ao ceticismo em Hessen (1980). Em Hjørland (2003) e Sudin e Johannisson (2005) também pode-se verificar que o pragmatismo filosófico é associado ao antirrealismo.

Podemos dizer que ainda não existe um consenso se a postura pragmática na OC é realista e se a postura positivista é antirrealista, conforme procuramos mostrar, ambas possuem aspectos semelhantes ao idealismo (antirrealismo), a primeira com o idealismo objetivo e a segunda com o idealismo subjetivo.

Não localizamos qualquer menção à Kant em Hjørland (2003, 2004), Sudin e Johannisson (2005) ou em demais pesquisas que abordam o debate sobre realismo e antirrealismo na OC.

Além disso, podemos dizer que o debate sobre realismo e antirrealimo tem recebido pouca atenção na $\mathrm{OC}$, com poucas pesquisas que abordam tal temática. Sudin e Johanisson (2005, p.39), afirmam ainda que

contentions between realism and anti-realism will not be solved with the help of criteria developed within the scientific community. We claim that the ontological issue of whether reality exists or not is seldom fruitful in LIS or other social sciences. 
Ainda que o debate sobre realismo e antirrealismo não possa ser resolvido com a ajuda de critérios desenvolvidos dentro da comunidade científica de $\mathrm{Cl}$ ou de outras Ciências Sociais, acreditamos ser imprudente ignorá-lo, ou ignorar as contribuições que pensadores, como Immanuel Kant, trouxeram a tal debate.

Um exemplo da relevância desse debate para a OC é apontado por Legg (2007, p. 425):

\begin{abstract}
A final issue worth mentioning-and it is an understatement to say that it has bedeviled philosophical ontology from the beginning-is the question of the degree to which the categories devised by human ontologists should be thought of as universally applicable or objective, as opposed to artifacts of particular contexts (such as a culture, a time period, a species, or a set of perceptual capacities). To what extent should an ontologist's categories be regarded as valid for all time and shareable across all communities? Or must all ontological work be done "locally" and repeatedly? This is the question of realism; 2,000 years of continuing controversy have not discouraged philosophers from hotly debating it today.
\end{abstract}

Com o intuito de identificar pesquisas em $O C$ que abordam o debate sobre realismo e antirrealismo realizou-se busca na base de dados Library and Information Science Abstracts (LISA), com estratégia de busca [(su(realism ) OR ti(realism ) OR ab(realism )) AND "Knowledge Organization"]. Foi possível localizar as seguintes pesquisas: Ducheyne (2009), Hartel e Hjorland (2003), Hjorland (2004), Mazzocchi (2013), Tomás Stodola (2014), Wild, Giess e Mcmahon (2009). No entanto, não identificamos referência ao pensamento de Kant em tais pesquisas.

\title{
2.3 Kant na pesquisa em Organização do Conhecimento
}

Conforme descrevemos ao longo desse trabalho, $\mathrm{O}$ pensamento de Immanuel Kant é importante para o debate epistemológico sobre o realismo e o antirrealismo ou idealismo e tal debate é importante para a pesquisa em OC. Como não localizamos referência ao pensamento de Kant nas pesquisas que abordam tal debate na OC e dada a importância de tal filósofo, optou-se pela busca de pesquisas em $\mathrm{OC}$ sobre o pensamento de Kant ou que se fundamentem nele.

Para isso, realizou-se busca na base de dados LISA com a estratégia de busca [(su(Kant ) OR ti(Kant ) OR ab(Kant )) AND "Knowledge Organization"], com a 
qual foi possível identificar três trabalhos Aranalde (2009), Barité (2000) e Amirhosseini (2010).

De modo geral, o foco das pesquisas de Aranalde (2009) e Barité (2000) são os princípios classificatórios e o estudo das categorias para Sistemas de Organização do Conhecimento. Amirhosseini (2010) fundamenta sua metodologia para a avaliação quantitativa de tesauros no pensamento de Kant.

$\mathrm{Na}$ pesquisa de Aranalde (2009) desenvolve-se uma reflexão sobre o processo de organização da informação e do conhecimento e os princípios classificatórios. Identifica as relações entre as categorias apresentadas em dois sistemas filosóficos de classificação - o aristotélico e o kantiano -, juntamente com as categorias formuladas pelo bibliotecário indiano Shiyali Ramamrita Ranganathan para orientar a identificação, classificação e organização do conhecimento registrado. As categorias foram identificadas como essenciais para a elaboração dos respectivos sistemas e para a elucidação de questões relativas ao conhecimento humano, seja de uma perspectiva que pretenda abarcar a totalidade da realidade, como no caso de Aristóteles, ou as condições da cognição humana, como no caso de Kant.

Aranalde (2009, p.94) aponta que Kant “tentou sintetizar os vários padrões de conhecimento existentes na época, que identificou como racionalismo, empirismo, idealismo e realismo." $\mathrm{E}$ descreve as principais diferenças entre as concepções kantianas e aristotélicas

o pensador alemão reconhece a importância e a grandiosidade do trabalho desenvolvido pelo estagirita, mas a análise de Aristóteles o coloca ora na classe dos realistas, ora na classe dos empiristas, indicando que qualquer uma das duas posições contém certa dose de ingenuidade. A explicação kantiana é que os empiristas reduzem todo conhecimento à experiência sensível. Por seu turno, os realistas acreditam num acesso imediato e direto aos objetos do conhecimento. Kant critica ambas as concepções, estabelecendo critérios para o conhecimento e concebendo seus objetos como coisas em si e fenômenos (ARANALDE, 2009, p.95)

A pesquisa de Barité (2000) também aborda a noção de categorias em Aristóteles, Kant e Ranganathan. De acordo com o autor,

The notion of category, from Aristotle and Kant to the present time, has been used as a basic intellectual tool for the analysis of the existence and 
changeableness of things. Ranganathan was the first to extrapolate the concept into the Theory of Classification, placing it as an essential axis for the logical organization of knowledge and the construction of indexing languages. This paper proposes a conceptual and methodological reexamination of the notion of category from a functional and instrumental perspective, and tries to clarify the essential characters of categories in that context, and their present implications regarding the construction and evaluation of indexing languages. (BARITÉ, 2000, p.4)

A pesquisa de Amirhosseini (2010) tem como base para a avaliação quantitativa de tesauros a epistemologia presente na obra 'A crítica da razão pura' de Kant.

we intend to suggest the complement ratio in the unity quantitative evaluation in a thesaurus term network. These ratios are the integration and relativeness ratios, which are connectedness ratio and complement ratio. The construction method of ratios is derived from Kant's philosophy in Critique of Pure Reason (1871). (AMIRHOSSEINI, 2010, p.186)

Amirhosseini (2010) também descreve a noção de espaço e tempo em Kant, as categorias Kantianas e a sua tentativa de mediar o empirismo de Hume e o racionalismo de Leibnitz e Wolff.

Não localizamos referência direta ou uma preocupação com o debate sobre realismo e idealismo em Barité (2000) e Amirhosseini (2010). Aranalde (2009), faz referência ao realismo ao apresentar a opinião de Kant sobre o pensamento de Aristóteles, mas não traz essa discussão para o âmbito da OC.

Podemos dizer, contudo, que ao desenvolver seus sistemas de categorias, tanto Aristóteles quanto Kant buscavam elucidar questões relativas ao conhecimento humano. Mas enquanto em Aristóteles essa busca parte do objeto, da realidade a ser conhecida, em Kant parte do sujeito que conhece e suas capacidades de apreender a realidade. Pois para Kant a apreensão da realidade por parte do sujeito e só é possível a partir de características que são inerentes ao próprio sujeito. E dessa forma, a coisa em si, como Kant denomina, ou uma realidade totalmente objetiva, não é acessível ao homem na visão de Kant.

Ao nos posicionarmos a favor dessa visão não estamos, contudo, advogando ou sugerindo que o sistema de categorias desenvolvido por Kant [3], que é discutido em Aranalde (2009) e Barité (2000), possa ser o mais efetivo para os propósitos da OC. Mas sim que ignorar a visão de Kant sobre o tema do realismo é 
no mínimo imprudente. Pois teorias, métodos e sistemas da $\mathrm{OC}$ que advogam e se fundamentam a partir de uma visão realista ingênua podem possuir um maior apelo, ao, por exemplo, serem declarados como objetivos e neutros, mas suas limitações têm sido cada vez mais evidenciadas na literatura da área.

\section{Considerações Finais}

De acordo com a discussão apresentada, podemos dizer que uma terceira via para a dicotomia realismo versus idealismo na OC seria bem vinda. Pois, essa dicotomia da teoria epistemológica se reflete na pesquisa em OC. Dois exemplos desse reflexo, que estão naturalmente ligados, é o debate sobre abordagem ontológica e epistemológica e postura positivista e pragmática na OC. Nesse sentido, o pensamento de Immanuel Kant, em especial sua filosofia transcendental presente na obra 'A crítica da razão pura' pode ser considerada como opção para fundamentar as pesquisas em OC. Em tal obra, Kant busca conciliar o realismo com o idealismo, igualmente como o fez entre o racionalismo e o empirismo. Assim como Gnoli (2008) sugere para as abordagens ontológica e epistemológica na OC.

Em pesquisas de cunho epistemológico na OC que abordam a temática do debate sobre realismo e idealismo ou antirrealismo não localizamos referência ao pensamento de Immanuel Kant. O próprio debate sobre realismo e idealismo é pouco estudado na OC e Cl. E Sudin e Johanisson (2005) consideram tal debate infrutífero na área pois consideram que tais questões não podem ser resolvidas a partir dos critérios das Ciências Sociais.

No entanto, entendemos que a dificuldade ou até a impossibilidade de uma solução para tal debate não diminui sua importância para o campo de OC. E nesse sentido, levar em conta todas as correntes teóricas existentes nos estudos epistemológicos nos parece prudente. Sendo que a área de epistemologia é a mais qualificada para abordar tal tema.

\section{Notas}

[1] De acordo com Hessen (1980, p.79) o apriorismo de Kant tem como intenção mediar o racionalismo de Leibnitz e Wolff e o empirismo de Locke e Hume. 
[2] Hjørland e Hartel (2003, p.243 e 244), de modo semelhante à Gnoli (2008), também diferenciam a natureza das teorias em ontológica e epistemológica, mas adicionam ainda a natureza sociológica e afirmam que "Human knowledge is thus a product of both the world itself and of human interests and capacities. It may be the case that different kinds of sciences may be more influenced by either the world or by human interests".

[3] Pois, conforme demonstra Umberto Eco, em seu livro Kant e o Ornitorrinco, no qual trata de problemas filosóficos diretamente relacionadas à OC - Como reconhecemos um gato? Por que o diferenciamos de um cachorro? Por que não confundimos um elefante com um tatu ou não chamamos uma mulher de chapéu? - que vem obcecando os pensadores desde Platão até os filósofos contemporâneos, e nem mesmo Kant soube solucioná-los em termos satisfatórios.

\section{Referências}

AMIRHOSSEINI, M. Theoretical Base of Quantitative Evaluation of Unity in a Thesaurus Term Network Based on Kant's Epistemology. Knowledge Organization, v. 37, n. 3, p. 185-202, 02010.

ARANALDE, M.M. Reflections on the Categorical Systems of Aristotle, Kant and Ranganathan. Ciencia da Informacao, v. 38, n. 1, p. 86-108, 02009.

BARITE, M.G. The notion of 'category': its implications in subject analysis and in the construction and evaluation of indexing languages. Knowledge Organization, v. 27, n. 1, p. 4-10, 02000.

DUCHEYNE, S. "To treat of the world". Journal of Documentation, Bradford, v. 65, n. 2, p. 223-244, 2009.

FRANCELIN, M. M; KOBASHI, N. Concepções sobre o conceito na organização da informação e do conhecimento. Ciência da Informação, Brasília, v. 40 n. 2, 2011.

GNOLI, C. Ten Long-Term Research Questions in Knowledge Organization. Knowledge Organization, v.35, n. 2, p.137-149, 2008.

HARTEL, J.; HJORLAND, B. Afterword: ontological, epistemological and sociological dimensions of domains. Knowledge Organization, v. 30, n. 3, p. 239-245, 02003.

HESSEN, Johannes. Teoria do conhecimento. 7. ed. Coimbra: A. Amado, 1980. 206p.

HJORLAND, B. Arguments for philosophical realism in library and information science. Library Trends, v. 52, n. 3, p. 488-506, 012004.

HJ ØRLAND, B. Fundamentals of Knowledge Organization. Knowledge Organization, v.30, n.2, p.87-111, 2003.

HJØRLAND, B. Semantic and Knowledge organization. Annual Review of Information Science and Technology, v.41, p. 367-405, 2007. 
HJØRLAND, B. ; CHRISTENSEN, F. S. Work Tasks and Socio-Cognitive Relevance:A Specific Example. JASIST, v.53, n.11, p.960-965, 2002.

HJØRLAND, B; HARTEL, J. Afterword: Ontological, Epistemological and Sociological Dimensions of Domains. Knowledge Organization, v. 30, n.3/4, 2003

HÖFFE, Otfried. Immanuel Kant. São Paulo: M. Fontes, 2005.

LEGG, C. Ontologies on the Semantic Web. Annual Review of Information Science and Technology, v. 41, n. 1, p. 407-451, 2007.

MAZZOCCHI, F. Images of Thought and Their Relation to Classification: The Tree and the Net. Knowledge Organization, v. 40, n. 6, p. 366-374, 02013.

MELO, M. A. F.; BRÄSCHER, M. Termo, conceito e relações conceituais: um estudo das propostas de Dahlberg e Hjorland. Ciência da Informação. 43, 1, 67-80, Jan. 2014.

MULLER, Robin, M. Kant and Saussure. Rivista Italiana di Filosofia del Linguaggio. v.3, 2010.

NORRIS, Christopher. Epistemologia: conceitos-chave em filosofia . Porto Alegre: Artmed, 2007. viii, 228 p.

POLI, Roberto. Ontology for knowledge organization. In Green, Rebecca, ed., Knowledge organization and change: Proceedings of the Fourth In.ternational ISKO Conference, 15-18 July, 1996 . Washington, DC.

POLI, Roberto. Framing ontology. In Corazzon, Raul, ed., Ontology: A resource guide for philosophers. 1997. Available at http://www.formalontology .it/essays/framing.pdf.

SILVA, Jesiel S. Intersecções epistemológicas: a binaridade de Saussure, a usiologia de Aristóteles e o cientificismo de Kant. Anthesis: Revista de Letras e Educação da Amazônia Sul-Ocidental, v. 1, n.2, 2012.

SUNDIN, O.; JOHANNISSON, J. Pragmatism, neo-pragmatism and sociocultural theory: Communicative participation as a perspective in LIS. Journal of Documentation, v.61, n. 1 , 2005.

TOMÁS STODOLA, J. The concept of information and questions of users with visual disabilities. Journal of Documentation, Bradford, v. 70, n. 5, p. 782-800, 2014.

WEISS, L. C.; BRÄSCHER, M. Abordagens e paradigmas na Organização do Conhecimento. In: GUIMARÃES, J. A.C.; DODEBEI, V. Organização do conhecimento e diversidade cultural. Marília: ISKO-Brasil: FUNDEPE, 2015.

WILD, P.J.; GIESS, M.D.; MCMAHON, C.A. Describing engineering documents with faceted approaches. Journal of Documentation, Bradford, v. 65, n. 3, p. 420-445, 2009. 